\title{
Molecular and Morphological Characterization of near Isogenic Lines Developed for Major Abiotic Stresses of Rice (Oryza sativa L.)
}

\author{
B. Sudhakar Reddy* and M. Girija Rani
}

Andhra Pradesh Rice Research institute \& Regional Agricultural Research Station, Maruteru, West Godavari District Andhra Pradesh-534122, India

*Corresponding author

\section{A B S T R A C T}

In the present investigation efforts were made to identify the distinct, uniform and stable morphological characters and molecular markers between NILs developed for SUB1 in

Keywords

Submergence, Lodging, Salinity, Near isogenic lines

Article Info

Accepted:

20 December 2017

Available Online:

10 January 2018 background of Pushyami (MTU 1075), Amara (MTU 1064), SALTOL in Cotton dora sannalu (MTU 1010) and lodging resistance in Swarna (MTU 7029) and Indra (MTU 1061) and their respective recurring parents. Rice productivity is limited by major abiotic stresses. Incorporation of stress tolerance gene/Qtls into popular varieties is one of the breeding strategies to combat adverse effects of climate changes. Characterization of rice genotypes is necessary for identification and protection of varieties under PPV. Results of molecular characterization showed genetic diversity among 24 entries. PIC value ranged from 0.08 (RM 6006) to 0.70 (RM 2229). Cluster analysis grouped 24 entries into two distinct clusters at similarity coefficient of $33 \%$. The results revealed that grouping of clusters using molecular markers is in accordance with parental ancestry and morphological traits. Graphical genotyping revealed that genome recovery of recurring parent in developed NILs ranged from 75.1\% (MTU 2546A-12-18-1) to $96.4 \%$ (MTU 2336-70-46-25-44). Most of the agro morphological characters were found to be similar between near isogenic lines and respective recurrent parent.

\section{Introduction}

Rice production and productivity is limited by major abiotic stresses like submergence, salinity, lodging in coastal irrigated ecosystem. Unabated efforts of researches resulted identification of SublA for flash flood tolerance (Xu and Mackill 1996; Nandi et al., 1997; Toojinda et al., 2003, Xu et al., 2006), Saltol for seedling stage salinity tolerance (Gregorio, 1997), SCM2 for lodging resistance (Ookawa et al., 2010). Marker assisted breeding played a vital role in development of Near Isogenic Lines (NILs) for major abiotic stress like salinity, submergence into widely adopted varities (Hoque et al., 2015; Renu Singh et al., 2015; Girijarani et al., 2015b; Iftekharuddaula et al., 2016). There is a need to characterize rice varieties according to DUS (Distinctiveness, Uniformity and Stability) test guidelines prescribed by Protection of Plant Varieties and Farmers' Rights Act Authority to protect varieties and varietal identification. Agro morphological characterization provides 
descriptors to distinguish one genotype from other. Techniques, such as plant characterization have been successfully used in recent years to help in identifying elite individuals. It is an indispensable tool for selecting varieties or lines based on agronomical, morphological, genetic or physiological characters (Ndour, 1998).

DNA fingerprinting with molecular markers allows precise, objective and rapid cultivar identification, it has been proved to be an efficient tool for characterization and management (Chakravarthi and Naravaneni, 2006). Simple sequence repeat (SSR) markers have been widely used for genetic analysis and cultivar identification by virtue of their abundance, co-dominant inheritance, high polymorphism, reproducibility and ease of assay by polymerase chain reaction (PCR) (Kuleung et al., 2004). It is essential to build the fingerprinting database of the main commercial cultivars in the market for rapid and unambiguous cultivar identification (Zhu et al., 2012). Background selection is the process of using markers covering all chromosomes and to accelerate the recovery of the recurrent parent genome during backcrossing (Hospital and Charcosset, 1997).

Characterization of NILs in comparison with recurrent parent is necessary for identification and protection of variety. Present study aimed to characterize NILs developed for submergence, salinity and lodging resistance using morphological and molecular markers.

\section{Materials and Methods}

Experiment was carried out at Andhra Pradesh Rice Research Institute and Regional Agricultural Research Station (APRRI and RARS) of West Godavari, Maruteru during kharif 2016 for morphological characterization. Experimental material consisting of rice Near Isogenic Lines (NILs) along with their corresponding recipient and donar parents developed for submergence, lodging and salinity tolerance developed at APRRI and RARS, Maruteru, West Godavari District. The coding of the experimental material used for the present study was presented in the Table 1. Twenty four entries comprising of NILs developed for Sub1 (NIL 1 and NIL 2) in background of Pushyami, Amara (NIL 5, 6, 7) using donar Swarna Sub1. NILs developed by incorporation SCM2 confers lodging resistance in Swarna (NIL 9, 10, 11) and Cottondora Sannalu (NIL 24) using donar II 110-9-1-1-1, Indra (NIL 16, 17, 18) using donar BPT 2270 and NILs of Cottondora Sannalu for Saltol (NIL 19, 20, 21) using FL478 as donar.

Experiment was layout in completely randomized block design in 2 replications. 25 old seedlings were transplanted with spacing of $20 \mathrm{~cm}$ between rows and $15 \mathrm{~cm}$ between plants. Recommended dose of fertilizers 90:60:60 kg/ha was provided. Molecular characterization was carried out during Rabi 2016-17.

\section{DNA isolation and PCR assay}

The total genomic DNA was isolated from 25 days old leaf samples as per the protocol described by Zheng et al., (1995) with some modifications. The quality and quantity of DNA was estimated using ND8000 eightchannel spectrophotometer. 10ulPCR reaction mixture consists of $1 \mu \mathrm{l}$ of $10 \mathrm{X}$ buffer $(10 \mathrm{mM}$ TrisHCl (pH 8.3), $50 \mathrm{mM} \mathrm{KCl}, 1.5 \mathrm{mM}$ $\mathrm{MgCl}_{2}, 0.01 \%$ gelatin), $0.5 \mu \mathrm{l}$ of dNTPs (2.5 $\mathrm{m} \mathrm{M}$. $\left.\mathrm{L}^{-1}\right), 1 \mu \mathrm{l}(5 \mu$ molar) each of forward and reverse primers, $1 \mu \mathrm{l}$ Taq DNA polymerase $(0.5 \mathrm{U} / \mu \mathrm{l})$ (Banglore Genei Private Limited, Bangalore), $3 \mu \mathrm{l}$ of template DNA $(10 \mathrm{ng} / \mu \mathrm{l})$ and $2.5 \mu \mathrm{l}$ of sterilized distilled water. Amplification were performed using Eppendorf thermo cycler with the temperature profiles of initial denaturation at 
$94^{\circ} \mathrm{C}$ for $5 \mathrm{~min}$, denaturation at $94^{\circ} \mathrm{C}$ for 0.5 min, annealing at $55^{\circ} \mathrm{C}$ for $0.5 \mathrm{~min}$, extension at $72^{\circ} \mathrm{C}$ for $1.0 \mathrm{~min}$ and final extension for 7 min at $72^{\circ} \mathrm{C}$ for 35 cycles. The PCR amplicons were electrophoresed on $3 \%$ agarose gel stained with ethidium bromide $(10 \mathrm{mg} / \mathrm{ml})$ at 100volts for $1.5 \mathrm{hr}$ in $1 \mathrm{X}$ TBE buffer. A 100 bp ladder (Genei) was used for appropriate sizing of the products. The gel images were captured under UV light using syngene Ingenius geldoc system.

\section{Molecular data analysis for characterization}

Total of sixty nine polymorphic markers were used for characterization of NILs along with their respective parents. The DNA banding patterns obtained from SSR analysis for each primer were scored by visual observation.

The 0/1 matrix was used to calculate the genetic similarity to estimate all pair-wise differences in the amplification products for all entries. The genetic similarity between these plants was evaluated by calculating the Jaccard similarity coefficient. Similarity coefficients were used for cluster analysis using sub program of NTSYS-PC (Rohlf, 2000). The dendrogram was constructed by unweighted pair group method with arithmetic averages (UPGMA) sub programme of NTSYS-PC.

Polymorphic information content (PIC) was calculated, according to the method of Anderson et al., (1993).

$$
\mathrm{PIC}=1-\sum_{f=1}^{n} \mathrm{P} i j^{2}
$$

Where P $i j$ is the frequency of the $\mathrm{jth}$ allele for the ith marker, and is summed over $\mathrm{n}$ alleles. The calculation was based on the number of alleles per locus.

\section{Genome recovery percentage $\%$}

The twenty-four entries consist of NILs along with their parents were screened with identified polymorphic SSR markers to decipher the percentage of the recurrent parent genome recovered (RPG) using Graphical Genotyping 2.0 (Van Berloo, 2008). The background recovery was calculated by using formula (Sundaram et al., 2008). In the present study background selection was performed using 58 polymorphic markers between Pushyami and Swarna Sub1 to assess genome recovery percentage of Pushyami. Sixty two polymorphic markers were used in the present study for background selection between recurrent parent Amara and donor Swarna Sub1. Background selection was performed using 57 polymorphic markers between Swarna and donor II 110-9-1-1-1-1 to assess genome recovery percentage of Swarna. A total of 50 polymorphic markers are used to assess genome recovery percentage of Indra. A total of 65 polymorphic markers are used to assess the genome recovery of Cottondora sannalu. Background selection was performed using 46 polymorphic markers between Cottondora sannalu and FL 478 to assess genome recovery of Cottondora sannalu.

$\mathrm{G}=[(\mathrm{X}+1 / 2 \mathrm{Y}) / 100] / \mathrm{N}$

$\mathrm{N}=$ total number of parental polymorphic markers screened

$\mathrm{X}=$ number of markers showing homozygosity for recurrent parent allele

$\mathrm{Y}=$ number of markers showing heterozygosity for parental alleles

\section{Characterization based on morphological traits}

The characteristics and their state and stage of observation were given as per the National 
Test Guidelines for Distinctness, Uniformity and Stability (DUS) (Shobarani et al., 2006) were recorded at different stages of crop growth period.

\section{Results and Discussion}

A total of 69 SSR markers were used covering all the chromosomes of rice for their molecular characterization and discrimination of twenty four entries of rice. The number of alleles per locus generated by each marker ranged from 1 to 4 alleles with an average number of 2.36 alleles per locus. The highest number of alleles (4) was detected for markers RM 243, RM 2972, RM 5210, RM 2229 and the lowest number of alleles (2) was detected for most of the primers. List of polymorphic microsatellite markers with their chromosomal locations, number of alleles and PIC value are presented in Table 2. The polymorphism information content (PIC) value ranged from 0.08 (RM 6006, RM 5055, RM 6364 and RM 2851 ) to 0.70 (RM 2229) with an average PIC of 0.41 .

Islam et al., (2012) observed a range of PIC value from 0.21 to 0.76 with an average of 0.57 in fourteen stress tolerant rice varieties of Bangladesh using 40 SSR primers and also revealed that higher the PIC value of a marker indicates higher probability of detecting the number of alleles among the cultivars. Markers with PIC values of 0.5 or higher are highly informative for genetic studies and are extremely useful in distinguishing the polymorphism rate of a marker at a specific locus (Virk et al., 1995). Markers with high PIC RM 5919, RM 212 and RM 2229 found to be useful in distinguishing NILs in present study.

\section{Cluster analysis}

Jaccard's coefficient value ranged from 0.212 to 1.00 among 24 entries studied (Table 3).
Near Isogenic Lines MTU 2547A-78-19-1-1 and MTU 2547A-77-11-1 showed $100 \%$ similarity. Genotypes with low similarity values are more divergent. Among the 24 entries FL 478 is more divergent with NILs of Pushyami with lower similarity value of 0.273, Sub1 donor Swarna Sub1 (NIL 4) also divergent with recurrent parent Pushyami (NIL 3), Amara (NIL 8) and NIL of Amara (NIL 6) with lower similarity value of 0.235 , 0.389 and 0.212 respectively. Swarna expressed lower similarity index with MTU 2244-119-59-63-40 (0.248), MTU 2244-11983-65 (0.235) NILs of Amara and recurrent parent Amara (0.263). MTU 2546A-34-1-9-1, a NIL of Swarna expressed lower similarity value of 0.270 with DST 8-162-4 NIL of Cottondora sannalu for Saltol. MTU 2251A136-11-1 NIL of Cottondora sannalu expressed lower similarity value of 0.265 with Swarna, II 110-9-1-1-1-1 (0.265), Indra (0.299), Bavapuri sannalu (0.263), it indicates these lines expressed diversity at molecular level.

Similar results were reported by Venuprasad et al., (2011) studied polymorphism analysis with 491 SSRs revealed that two NIL pairs are at least $96 \%$ genetically similar. Further the results were in agreement with, Patel et al., (2016) conducted molecular characterization of Near Isogenic Lines using 29 RAPD and SSR markers.

A dendrogram (Figure 1) indicated that there were two major clusters 'I' and 'II' at 33\% similarly level. Major cluster 'I' divided into two sub cluster IA with 8 entries and IB with 10 entries. NILs of Pushyami (MTU 2336-6225-38-16 and MTU 2336-70-46-25-44) and recurrent parent Pushyami (MTU1075), Swarna and NILs of Swarna for lodging resistance (MTU 2546A-13-1-6-1, MTU 2546A-12-18-1, MTU 2546A-34-1-9-1) and Swarna Sub1 were grouped in cluster IA. The cluster IB consist of Amara and its Sub1 NILs 
(MTU 2244-119-59-63-40, MTU 2244-11983-65, MTU 2241-39-10-44-1), Indra along with its NILs for lodging resistance (MTU 2547A-78-19-1-1, MTU 2547A-77-11-1, MTU 2547A-95-1-11-1), Bhavapuri sannalu and II 110-9-1-1-1-1 (Table 4). Major cluster 'II' divided into two sub cluster IIA with 5 entries and IIB with one entry. Cluster IIA consisted of Cottondora sannalu along with NILs for salinity tolerance (DST 8-162-4, DST 9-157-7, and DST 8-4-4) and a NIL for lodging (MTU 2251A-136-11-1). IIB consists of FL 478, a saltol donor.

Cluster IA consists of Swarna and its derived lines and Pushyami and its derived lines. Grouping of this cluster clearly indicated that Pushyami and Swarna have genome relationship in their ancestry because Swarna was derived from Vasista and Mashuri. Pushyami was derived from MTU 2716 and MTU 1010. While MTU 2716 was developed from Mashuri and Vijaya. This clearly demonstrated that Mashuri parent is common ancestry parent has contributed maximum genome inheritance in the development of Swarna and Pushyami and their respective Near Isogenic Lines.

Whereas cluster IB comprises most of Indra and Amara derived lines. It clearly indicated that Indra and Amara have same parents of PLA 1100 and MTU 1010. PLA 1100 intern derived from Mashuri and Vijaya. Bavapuri sannalu is derived from BPT 5204 and CR15MR1523 and BPT 5204 has Mashuri as one of the parent. II 110-9-1-1-1-1 derived from a cross between [(BPT 5204 / IET 9762)/ Swarna]/MTU2716 in which the parents BPT 5204, Swarna, MTU 2716 has common parent of Mashuri. Entries grouped in cluster I are late in duration with intermediate height. Cluster IIA consists of MTU 1010 and its derived lines and IIB consist of FL 478, donor for salinity tolerance. All the lines are dwarf and early in duration.
The above results revealed that grouping of cluster I using molecular markers is in accordance with parental ancestry and morphological traits. This indicated that markers used in this study can be useful to distinguish entries studied.

\section{Genome recovery percentage}

The results of graphical genotyping revealed that genome recovery percentage of NILs ranged from 75.1\% (MTU 2546A-12-18-1) to $96.4 \%$ (MTU 2336-70-46-25-44). The genome recovery percentages of NILs were presented in the Table 5.

NIL MTU 2336-70-46-25-44 showed highest genome recovery percentage of $96.4 \%$ among Subl introgressed lines of Pushyami.

NILs of Amara, MTU 2244-119-59-63-40 and MTU 2244-119-83-65 showed highest genome recovery percentage of $96.3 \%$ and $96.2 \%$ respectively among the Subl introgressed lines of Amara.

It indicates that this NILs are better recovered from respective recurrent parent Pushyami and Amara with Subl locus. These NILs would be adopted by farmers in flood prone areas after intensive evaluation and testing.

Similar results were reported by Renu singh et al., (2015) in 18 advanced backcross lines (M1-M17 and M20) from MTU 1075/Swarna-Sub1 cross at $\mathrm{BC}_{3} \mathrm{~F}_{4}$ generation, showed $91.88-90.29 \%$ overall recipient genome recovery and $85.35-88.45 \%$.

Three best plants were selected and their recipient genome recovery percentages were $86.84 \%, 85.13 \%$ and $85.0 \%$ in Sub1 lines of BRRI dhan49 (Ara et al., 2015). Ahmed et al., (2016) background analysis of Sub1 incorporated lines of MR219 revealed genome recovery of $95.37 \%$ at $\mathrm{BC}_{2} \mathrm{~F}_{2}$ generations. 
Table.1 Experimental material used for characterization during kharif and rabi 2016-17

\begin{tabular}{l|l|l|}
\hline CODE & \multicolumn{1}{|c|}{ DESIGNATION } & \multicolumn{1}{c}{ CROSS COMIBINATION } \\
\hline NIL 1 & MTU 2336-62-25-38-16 & MTU 1075/SWARNA SUB1//*3 MTU 1075 \\
NIL 2 & MTU 2336-70-46-25-44 & MTU 1075/SWARNA SUB1//*3 MTU 1075 \\
\hline NIL 3 & Pushyami (MTU 1075) & RECURRENT PARENT \\
NIL 4 & SWARNA SUB1 & DONAR PARENT \\
NIL 5 & MTU 2244-119-59-63-40 & MTU 1064/SWARNA SUB1//*3 MTU 1064 \\
NIL 6 & MTU 2244-119-83-65 & MTU 1064/SWARNA SUB1//*3 MTU 1064 \\
NIL 7 & MTU 2244-39-10-44-1 & MTU 1064/SWARNA SUB1//*3 MTU 1064 \\
\hline NIL 8 & Amara (MTU 1064) & RECURRENT PARENT \\
NIL 9 & MTU 2546A-13-1-6-1 & MTU 7029/II 110-9-1-1-1-1//*3 MTU 7029 \\
NIL 10 & MTU 2546A-12-18-1 & MTU 7029/II 110-9-1-1-1-1//*3 MTU 7029 \\
\hline NIL 11 & MTU 2546A-34-1-9-1 & MTU 7029/II 110-9-1-1-1-1//*3 MTU 7029 \\
\hline NIL 12 & Swarna (MTU 7029) & RECURRENT PARENT \\
NIL 13 & II 110-9-1-1-1-1 & DONAR PARENT \\
NIL 14 & Indra (MTU 1061) & RECURRENT PARENT \\
NIL 15 & Bavapuri sannalu (BPT 2270) & DONAR PARENT \\
NIL 16 & MTU 2547A-78-19-1-1 & MTU 1061/BPT 2270//*3 MTU 1061 \\
\hline NIL 17 & MTU 2547A-77-11-1 & MTU 1061/BPT 2270//*3 MTU 1061 \\
NIL 18 & MTU 2547A-95-1-11-1 & MTU 1061/BPT 2270//*3 MTU 1061 \\
\hline NIL 19 & DST 8-162-4 & MTU 1010/FL 478//*3 MTU 1010 \\
NIL 20 & DST 9-157-7 & MTU 1010/FL 478//*3 MTU 1010 \\
\hline NIL 21 & DST 8-4-4 & MTU 1010/FL 478//*3 MTU 1010 \\
NIL 22 & FL 478 & DONAR PARENT \\
\hline NIL 23 & Cottondora sannalu (MTU 1010) & RECURRENT PARENT \\
NIL 24 & MTU 2251A-136-11-1 & MTU 1010/ II 110-9-1-1-1-1//*3 MTU 1010 \\
\hline & &
\end{tabular}

Table.2 List of polymorphic microsatellite markers among 24 rice entries

\begin{tabular}{|c|c|c|c|c|}
\hline S. No. & SSR Markers & Chromosomal number & Number of alleles & PIC Value \\
\hline 1 & RM 243 & 1 & 4 & 0.64 \\
\hline 2 & RM 5919 & 1 & 3 & 0.66 \\
\hline 3 & RM 212 & 1 & 3 & 0.66 \\
\hline 4 & RM 246 & 1 & 2 & 0.50 \\
\hline 5 & AP 3206 & 1 & 2 & 0.50 \\
\hline 6 & RM 10694 & 1 & 2 & 0.38 \\
\hline 7 & RM 3412 & 1 & 2 & 0.15 \\
\hline 8 & RM 10855 & 1 & 3 & 0.66 \\
\hline 9 & RM 6334 & 1 & 2 & 0.33 \\
\hline 10 & RM 3865 & 2 & 3 & 0.54 \\
\hline 11 & RM 106 & 2 & 2 & 0.47 \\
\hline 12 & RM 5210 & 2 & 4 & 0.60 \\
\hline 13 & RM 3340 & 2 & 2 & 0.49 \\
\hline 14 & RM 6933 & 2 & 3 & 0.47 \\
\hline 15 & RM 1230 & 3 & 2 & 0.28 \\
\hline 16 & RM 5924 & 3 & 3 & 0.60 \\
\hline 17 & RM 1350 & 3 & 2 & 0.22 \\
\hline 18 & RM 5474 & 3 & 2 & 0.15 \\
\hline 19 & RM 3524 & 4 & 2 & 0.47 \\
\hline
\end{tabular}


Int.J.Curr.Microbiol.App.Sci (2018) 7(1): 2782-2797

\begin{tabular}{|c|c|c|c|c|}
\hline 20 & RM 335 & 4 & 3 & 0.49 \\
\hline 21 & RM 303 & 4 & 3 & 0.55 \\
\hline 22 & RM 241 & 4 & 2 & 0.50 \\
\hline 23 & RM 6006 & 4 & 2 & 0.08 \\
\hline 24 & RM 169 & 5 & 2 & 0.49 \\
\hline 25 & RM 249 & 5 & 2 & 0.22 \\
\hline 26 & RM 163 & 5 & 2 & 0.47 \\
\hline 27 & RM 6024 & 5 & 2 & 0.08 \\
\hline 28 & RM 8107 & 6 & 2 & 0.49 \\
\hline 29 & RM 3 & 6 & 2 & 0.54 \\
\hline 30 & RM 225 & 6 & 2 & 0.44 \\
\hline 31 & RM 19382 & 6 & 2 & 0.44 \\
\hline 32 & RM 2229 & 6 & 4 & 0.70 \\
\hline 33 & RM 253 & 6 & 2 & 0.29 \\
\hline 34 & RM 20547 & 6 & 2 & 0.28 \\
\hline 35 & RM 5509 & 6 & 3 & 0.63 \\
\hline 36 & RM 20557 & 6 & 2 & 0.33 \\
\hline 37 & RM 340 & 6 & 2 & 0.38 \\
\hline 38 & RM 23865 & 6 & 3 & 0.57 \\
\hline 39 & RM 20648 & 6 & 3 & 0.52 \\
\hline 40 & RM 464 & 6 & 2 & 0.49 \\
\hline 41 & RM 23805 & 6 & 2 & 0.44 \\
\hline 42 & RM 23869 & 6 & 2 & 0.22 \\
\hline 43 & Sub1BC2 & 6 & 2 & 0.22 \\
\hline 44 & RM 30 & 6 & 2 & 0.38 \\
\hline 45 & RM 320 & 7 & 3 & 0.60 \\
\hline 46 & RM 478 & 7 & 2 & 0.15 \\
\hline 47 & RM 5055 & 7 & 2 & 0.08 \\
\hline 48 & RM 5720 & 7 & 3 & 0.57 \\
\hline 49 & RM 149 & 8 & 2 & 0.33 \\
\hline 50 & RM 1111 & 8 & 2 & 0.44 \\
\hline 51 & RM 566 & 9 & 3 & 0.63 \\
\hline 52 & RM 524 & 9 & 3 & 0.52 \\
\hline 53 & RM 528 & 9 & 2 & 0.22 \\
\hline 54 & RM 23915 & 9 & 2 & 0.33 \\
\hline 55 & RM 23788 & 9 & 2 & 0.22 \\
\hline 56 & RM 6100 & 10 & 2 & 0.50 \\
\hline 57 & RM 484 & 10 & 2 & 0.15 \\
\hline 58 & RM 6364 & 10 & 2 & 0.08 \\
\hline 59 & RM 5926 & 11 & 2 & 0.38 \\
\hline 60 & RM 286 & 11 & 2 & 0.41 \\
\hline 61 & RM 6925 & 11 & 3 & 0.53 \\
\hline 62 & RM 5766 & 11 & 2 & 0.28 \\
\hline 63 & RM 6293 & 11 & 2 & 0.50 \\
\hline 64 & RM 2972 & 12 & 4 & 0.58 \\
\hline 65 & RM 309 & 12 & 2 & 0.49 \\
\hline 66 & RM 5939 & 12 & 3 & 0.58 \\
\hline 67 & RM 1227 & 12 & 2 & 0.41 \\
\hline 68 & RM 2529 & 12 & 2 & 0.49 \\
\hline \multirow[t]{2}{*}{69} & RM 2851 & 12 & 2 & 0.08 \\
\hline & Average & & 2.36 & 0.41 \\
\hline
\end{tabular}


Table.3 Jaccard's similarity coefficient matrix of 24 entries

\begin{tabular}{|c|c|c|c|c|c|c|c|c|c|c|c|c|c|c|c|c|c|c|c|c|c|c|c|c|}
\hline & NIL1 & NIL2 & NIL3 & NIL4 & NIL5 & NIL6 & NIL7 & NIL8 & NIL9 & NIL10 & NIL11 & NIL12 & NIL13 & NIL14 & NIL15 & NIL16 & NIL17 & NIL18 & NIL19 & NIL20 & NIL21 & NIL22 & NIL23 & NIL24 \\
\hline NIL1 & 1.000 & & & & & & & & & & & & & & & & & & & & & & & \\
\hline NIL2 & 0.775 & 1.000 & & & & & & & & & & & & & & & & & & & & & & \\
\hline NIL3 & 0.703 & 0.658 & 1.000 & & & & & & & & & & & & & & & & & & & & & \\
\hline NIL4 & 0.385 & 0.416 & 0.235 & 1.000 & & & & & & & & & & & & & & & & & & & & \\
\hline NIL5 & 0.355 & 0.326 & 0.432 & 0.248 & 1.000 & & & & & & & & & & & & & & & & & & & \\
\hline NIL6 & 0.313 & 0.340 & 0.416 & 0.212 & 0.853 & 1.000 & & & & & & & & & & & & & & & & & & \\
\hline NIL7 & 0.400 & 0.400 & 0.465 & 0.260 & 0.595 & 0.615 & 1.000 & & & & & & & & & & & & & & & & & \\
\hline NIL8 & 0.302 & 0.302 & 0.389 & 0.214 & 0.761 & 0.838 & 0.689 & 1.000 & & & & & & & & & & & & & & & & \\
\hline NIL9 & 0.416 & 0.416 & 0.448 & 0.400 & 0.370 & 0.355 & 0.615 & 0.437 & 1.000 & & & & & & & & & & & & & & & \\
\hline NIL10 & 0.340 & 0.432 & 0.416 & 0.400 & 0.340 & 0.355 & 0.537 & 0.389 & 0.615 & 1.000 & & & & & & & & & & & & & & \\
\hline NIL11 & 0.355 & 0.385 & 0.340 & 0.595 & 0.260 & 0.248 & 0.400 & 0.276 & 0.556 & 0.615 & 1.000 & & & & & & & & & & & & & \\
\hline NIL12 & 0.355 & 0.385 & 0.340 & 0.615 & 0.248 & 0.235 & 0.385 & 0.263 & 0.537 & 0.636 & 0.853 & 1.000 & & & & & & & & & & & & \\
\hline NIL13 & 0.292 & 0.363 & 0.363 & 0.255 & 0.278 & 0.319 & 0.430 & 0.295 & 0.398 & 0.482 & 0.352 & 0.337 & 1.000 & & & & & & & & & & & \\
\hline NIL14 & 0.340 & 0.340 & 0.313 & 0.248 & 0.416 & 0.500 & 0.556 & 0.506 & 0.465 & 0.370 & 0.273 & 0.260 & 0.430 & 1.000 & & & & & & & & & & \\
\hline NIL15 & 0.359 & 0.276 & 0.330 & 0.238 & 0.404 & 0.389 & 0.506 & 0.425 & 0.359 & 0.344 & 0.289 & 0.302 & 0.402 & 0.437 & 1.000 & & & & & & & & & \\
\hline NIL16 & 0.326 & 0.326 & 0.299 & 0.235 & 0.400 & 0.482 & 0.537 & 0.488 & 0.448 & 0.355 & 0.286 & 0.273 & 0.414 & 0.969 & 0.420 & 1.000 & & & & & & & & \\
\hline NIL17 & 0.326 & 0.326 & 0.299 & 0.235 & 0.400 & 0.482 & 0.537 & 0.488 & 0.448 & 0.355 & 0.286 & 0.273 & 0.414 & 0.969 & 0.420 & 1.000 & 1.000 & & & & & & & \\
\hline NIL18 & 0.370 & 0.370 & 0.340 & 0.273 & 0.385 & 0.432 & 0.518 & 0.437 & 0.465 & 0.340 & 0.326 & 0.313 & 0.398 & 0.881 & 0.404 & 0.909 & 0.909 & 1.000 & & & & & & \\
\hline NIL19 & 0.351 & 0.337 & 0.380 & 0.283 & 0.309 & 0.270 & 0.283 & 0.260 & 0.323 & 0.323 & 0.270 & 0.296 & 0.302 & 0.351 & 0.286 & 0.366 & 0.366 & 0.411 & 1.000 & & & & & \\
\hline NIL20 & 0.326 & 0.313 & 0.340 & 0.286 & 0.299 & 0.286 & 0.273 & 0.276 & 0.299 & 0.313 & 0.273 & 0.299 & 0.292 & 0.340 & 0.344 & 0.355 & 0.355 & 0.370 & 0.868 & 1.000 & & & & \\
\hline NIL21 & 0.400 & 0.385 & 0.416 & 0.299 & 0.313 & 0.326 & 0.340 & 0.316 & 0.340 & 0.340 & 0.313 & 0.340 & 0.348 & 0.355 & 0.316 & 0.370 & 0.370 & 0.416 & 0.814 & 0.775 & 1.000 & & & \\
\hline NIL22 & 0.273 & 0.273 & 0.273 & 0.260 & 0.313 & 0.326 & 0.340 & 0.330 & 0.370 & 0.313 & 0.299 & 0.299 & 0.292 & 0.370 & 0.330 & 0.385 & 0.385 & 0.400 & 0.512 & 0.595 & 0.518 & 1.000 & & \\
\hline NIL23 & 0.432 & 0.416 & 0.448 & 0.326 & 0.340 & 0.326 & 0.340 & 0.316 & 0.340 & 0.355 & 0.313 & 0.340 & 0.333 & 0.355 & 0.302 & 0.370 & 0.370 & 0.416 & 0.841 & 0.800 & 0.909 & 0.465 & 1.000 & \\
\hline NIL24 & 0.385 & 0.370 & 0.400 & 0.286 & 0.313 & 0.299 & 0.299 & 0.289 & 0.299 & 0.313 & 0.273 & 0.299 & 0.265 & 0.299 & 0.263 & 0.313 & 0.313 & 0.355 & 0.716 & 0.680 & 0.775 & 0.385 & 0.853 & 1.000 \\
\hline
\end{tabular}


Table.4 Clustering pattern of the rice entries based on molecular data

\begin{tabular}{|c|c|c|c|}
\hline $\begin{array}{l}\text { Main } \\
\text { cluster }\end{array}$ & $\begin{array}{l}\text { Sub } \\
\text { cluster }\end{array}$ & $\begin{array}{l}\text { No. of } \\
\text { entries }\end{array}$ & Designation of entries \\
\hline \multirow[t]{2}{*}{ I } & IA & 8 & $\begin{array}{l}\text { MTU2336-62-25-38-16, MTU2336-70-46-25-44, } \\
\text { MTU1075, MTU2546A-13-1-6-1, MTU2546A- } \\
\text { 12-18-1, MTU2546A-34-1-9-1, MTU7029, } \\
\text { Swarna Sub1. }\end{array}$ \\
\hline & IB & 10 & $\begin{array}{l}\text { MTU 2244-119-59-63-40, MTU 2244-119-83-65, } \\
\text { MTU 2241-39-10-44-1, MTU 1064, MTU 2547A- } \\
\text { 78-19-1-1, MTU 2547A-77-11-1, MTU 2547A-- } \\
\text { 95-1-11-1, MTU1061, BPT2270, II 110-9-1-1-1-1 }\end{array}$ \\
\hline \multirow[t]{2}{*}{ II } & IIA & 5 & $\begin{array}{l}\text { DST 8-162-4, DST 9-157-7, DST 8-4-4, MTU } \\
\text { 2251A-136-11-1, MTU1010. }\end{array}$ \\
\hline & IIB & 1 & FL 478. \\
\hline
\end{tabular}

Table.5 Genome recovery percentage of Near Isogenic Lines (NILs) developed for submergence, lodging and salinity

\begin{tabular}{|c|c|c|c|}
\hline S. No. & Entry & Designation & Genome recovery $\%$ \\
\hline 1 & NIL of Pushyami for Subl & MTU 2336-62-25-38-16 & $89.9 \%$ \\
\hline 2 & NIL of Pushyami for Subl & MTU 2336-70-46-25-44 & $96.4 \%$ \\
\hline 3 & Recurrent parent & Pushyami (MTU 1075) & \\
\hline 4 & Donar parent & Swarna Sub1 & \\
\hline 5 & NIL of Amara for Subl & MTU 2244-119-59-63-40 & $96.3 \%$ \\
\hline 6 & NIL of Amara for Subl & MTU 2244-119-83-65 & $96.2 \%$ \\
\hline 7 & NIL of Amara for $S u b 1$ & MTU 2244-39-10-44-1 & $86.4 \%$ \\
\hline 8 & Recurrent parent & Amara (MTU 1064) & \\
\hline 9 & NIL of Swarna for $S C M 2$ & MTU 2546A-13-1-6-1 & $79.3 \%$ \\
\hline 10 & NIL of Swarna for $S C M 2$ & MTU 2546A-12-18-1 & $75.1 \%$ \\
\hline 11 & NIL of Swarna for SCM2 & MTU 2546A-34-1-9-1 & $94.1 \%$ \\
\hline 12 & Recurrent parent & Swarna (MTU 7029) & \\
\hline 13 & Donar parent & II 110-9-1-1-1-1 & \\
\hline 14 & Recurrent parent & Indra (MTU 1061) & \\
\hline 15 & Donar parent & Bavapuri sannalu (BPT 2270) & \\
\hline 16 & NIL of Indra for $S C M 2$ & MTU 2547A-78-19-1-1 & $93.7 \%$ \\
\hline 17 & NIL of Indra for $S C M 2$ & MTU 2547A-77-11-1 & $88.4 \%$ \\
\hline 18 & NIL of Indra for $S C M 2$ & MTU 2547A-95-1-11-1 & $90.2 \%$ \\
\hline 19 & NIL of Cottondora sannalu for Saltol & DST 8-162-4 & $91.0 \%$ \\
\hline 20 & NIL of Cottondora sannalu for Saltol & DST 9-157-7 & $78.3 \%$ \\
\hline 21 & NIL of Cottondora sannalu for Saltol & DST 8-4-4 & $89.1 \%$ \\
\hline 22 & Donar parent & FL 478 & \\
\hline 23 & Recurrent parent & Cottondora sannalu (MTU 1010) & \\
\hline 24 & NIL of MTU 1010 for SCM2 & MTU 2251A-136-11-1 & $93.8 \%$ \\
\hline
\end{tabular}


Table.6 Summary results of morphological and abiotic stress tolerance among 24 entries of rice

\begin{tabular}{|c|c|c|c|c|c|}
\hline S. No. & $\begin{array}{l}\text { Recurrent } \\
\text { parent }\end{array}$ & $\begin{array}{l}\text { Targeted } \\
\text { trait }\end{array}$ & $\begin{array}{l}\text { Near Isogenic Lines of } \\
\text { recurrent parent }\end{array}$ & $\begin{array}{l}\text { Distinct morphological character from } \\
\text { recurrent parent }\end{array}$ & $\begin{array}{l}\text { Tolerance to other abiotic } \\
\text { stress }\end{array}$ \\
\hline \multirow[t]{2}{*}{1.} & \multirow[t]{2}{*}{$\begin{array}{l}\text { Pushyami } \\
\text { (MTU 1075) }\end{array}$} & \multirow[t]{2}{*}{$\begin{array}{l}\text { Submergence } \\
\text { tolerance }\end{array}$} & MTU 2336-62-25-38-16 & $\begin{array}{l}\text { 1. Dark green intensity of leaf colour } \\
\text { 2. Medium leaf senescence }\end{array}$ & $\begin{array}{l}\text { Stagnant flood tolerance and } \\
\text { lodging resistance }\end{array}$ \\
\hline & & & MTU 2336-70-46-25-44 & $\begin{array}{l}\text { 1. Dark green intensity of leaf colour } \\
\text { 2. Medium leaf senescence }\end{array}$ & $\begin{array}{l}\text { Stagnant flood tolerance and } \\
\text { lodging resistance }\end{array}$ \\
\hline \multirow[t]{3}{*}{2.} & \multirow[t]{3}{*}{$\begin{array}{l}\text { Amara } \\
(\text { MTU 1064) }\end{array}$} & \multirow[t]{3}{*}{$\begin{array}{l}\text { Submergence } \\
\text { tolerance }\end{array}$} & MTU 2244-119-59-63-40 & $\begin{array}{l}\text { 1. Weak pubescence of blade surface } \\
\text { 2. Medium leaf senescence } \\
\text { 3. Medium gelatinizing temperature }\end{array}$ & $\begin{array}{l}\text { Stagnant flood tolerance, lodging } \\
\text { resistance and moderate salinity } \\
\text { tolerance. }\end{array}$ \\
\hline & & & MTU 2244-119-83-65 & $\begin{array}{l}\text { 1. Weak pubescence of blade surface } \\
\text { 2. Long panicle length }(<25 \mathrm{~cm}) \\
\text { 3. Straw colour lemma and palea } \\
\text { 4. Presence of panicle awns } \\
\text { 5. Medium leaf senescence } \\
\text { 6. Very low } 1000 \text { grain weight }(<15 \mathrm{~g}) \\
\text { 7. Narrow grain and decorticated grain width. } \\
\text { 8. Medium gelatinizing temperature }\end{array}$ & $\begin{array}{l}\text { Stagnant flood tolerance, lodging } \\
\text { resistance and moderate salinity } \\
\text { tolerance. }\end{array}$ \\
\hline & & & MTU 2244-39-10-44-1 & $\begin{array}{l}\text { 1. Weak pubescence of blade surface } \\
\text { 2. Medium leaf senescence } \\
\text { 3. Medium gelatinizing temperature }\end{array}$ & $\begin{array}{l}\text { Stagnant flood tolerance, lodging } \\
\text { resistance and moderate salinity } \\
\text { tolerance. }\end{array}$ \\
\hline \multirow[t]{2}{*}{3.} & \multirow[t]{2}{*}{$\begin{array}{l}\text { Swarna } \\
\text { (MTU 7029) }\end{array}$} & \multirow[t]{2}{*}{$\begin{array}{l}\text { Lodging } \\
\text { resistance }\end{array}$} & MTU 2546A-13-1-6-1 & $\begin{array}{l}\text { 1. Medium pubescence of blade surface } \\
\text { 2. Thick stem thickness } \\
\text { 3. High medium gelatinizing temperature } \\
\text { 4. Medium leaf senescence }\end{array}$ & $\begin{array}{l}\text { Stagnant flood tolerance, } \\
\text { moderate tolerance to anaerobic } \\
\text { germination }\end{array}$ \\
\hline & & & MTU 2546A-12-18-1 & $\begin{array}{l}\text { 1. Medium pubescence of blade surface } \\
\text { 2. Thick stem thickness } \\
\text { 3. long panicle length } \\
\text { 4. High medium gelatinizing temperature } \\
\text { 5. Medium leaf senescence }\end{array}$ & $\begin{array}{l}\text { Stagnant flood tolerance, } \\
\text { moderate tolerance to Anaerobic } \\
\text { germination }\end{array}$ \\
\hline
\end{tabular}




\begin{tabular}{|c|c|c|c|c|c|}
\hline S.No. & $\begin{array}{l}\text { Recurrent } \\
\text { parent }\end{array}$ & $\begin{array}{l}\text { Targeted } \\
\text { trait }\end{array}$ & $\begin{array}{l}\text { Near Isogenic Lines of } \\
\text { recurrent parent }\end{array}$ & $\begin{array}{l}\text { Distinct morphological character from } \\
\text { recurrent parent }\end{array}$ & $\begin{array}{l}\text { Tolerance to other abiotic } \\
\text { stress }\end{array}$ \\
\hline \multirow{3}{*}{4.} & & & MTU 2546A-34-1-9-1 & $\begin{array}{l}\text { 1. Medium pubescence of blade surface } \\
\text { 2. Thick stem thickness } \\
\text { 3. Medium leaf senescence }\end{array}$ & $\begin{array}{l}\text { Moderate tolerance to anaerobic } \\
\text { germination }\end{array}$ \\
\hline & \multirow{2}{*}{$\begin{array}{l}\text { Indra } \\
(\text { MTU 1061) }\end{array}$} & \multirow{2}{*}{$\begin{array}{l}\text { Lodging } \\
\text { resistance }\end{array}$} & MTU 2547A-78-19-1-1 & No variation is observed & Moderate salinity tolerance \\
\hline & & & MTU 2547A-77-11-1 & 1. Medium 1000 grain weight & Anaerobic germination \\
\hline \multirow[t]{4}{*}{5.} & \multirow[t]{4}{*}{$\begin{array}{l}\text { Cottondora } \\
\text { sannalu } \\
\text { MTU (1010) }\end{array}$} & \multirow[t]{3}{*}{$\begin{array}{l}\text { Salinity } \\
\text { tolerance }\end{array}$} & DST 8-162-4 & $\begin{array}{l}\text { 1. Strong pubescence of blade surface } \\
\text { 2. Thick stem thickness } \\
\text { 3. Long panicle length } \\
\text { 4. Medium panicle number per plant }(>11)\end{array}$ & $\begin{array}{l}\text { Lodging resistance, Anaerobic } \\
\text { germination }\end{array}$ \\
\hline & & & DST 9-157-7 & $\begin{array}{l}\text { 1. Strong pubescence of blade surface } \\
\text { 2. Thick stem thickness } \\
\text { 3. Long panicle length } \\
\text { 4. Medium panicle number per plant }(>11) \\
\text { 5. High } 1000 \text { grain weight } \\
\text { 6. Medium grain and decorticated grain width. }\end{array}$ & $\begin{array}{l}\text { Lodging resistance, Anaerobic } \\
\text { germination, flash flood tolerance }\end{array}$ \\
\hline & & & DST 8-4-4 & $\begin{array}{l}\text { 1. Strong pubescence of blade surface } \\
\text { 2. Thick stem thickness } \\
\text { 3. Long panicle length } \\
\text { 4. Medium panicle number per plant }(>11) \\
\text { 5. High } 1000 \text { grain weight } \\
\text { 6. Medium grain and decorticated grain width. }\end{array}$ & $\begin{array}{l}\text { Lodging resistance, Anaerobic } \\
\text { germination, stagnant flood } \\
\text { tolerance }\end{array}$ \\
\hline & & $\begin{array}{l}\text { Lodging } \\
\text { resistance }\end{array}$ & MTU 2251A-136-11-1 & $\begin{array}{l}\text { 1. Strong pubescence of blade surface } \\
\text { 2. Thick stem thickness }\end{array}$ & \\
\hline
\end{tabular}


Fig.1 Dendogram depicting clustering pattern of the twenty four entries of rice using SSR markers. The scale at the bottom is Jaccard's similarity coefficient of genetic similarity

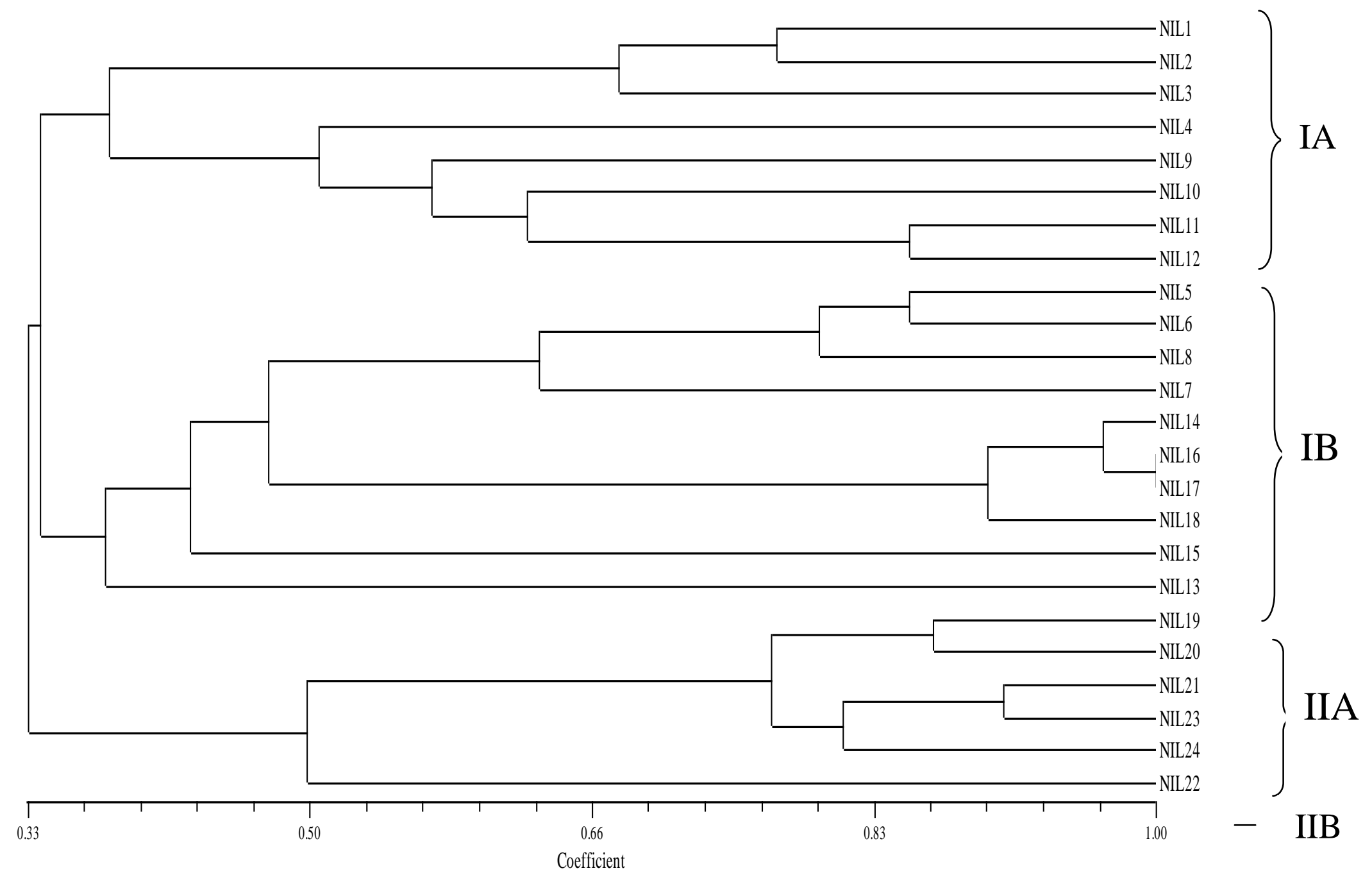


MTU 2546A-34-1-9-1 NIL of Swarna with non-lodging trait showed highest genome recovery of $94.1 \%$. NILs MTU 2547A-7819-1-1 and MTU 2547A-95-1-11-1 showed highest genome recovery $93.7 \%$ and $90.2 \%$ respectively for recurrent parent Indra. MTU 2251A-136-11-1, NIL of Cottondora sannalu showed genome recovery of $93.8 \%$.

This NILs with targeted trait of lodging in the background of highly adaptive varieties Swarna (MTU 7029), Cottondora sannalu (MTU 1010) and popular variety Indra (MTU 1061) would withstand adverse effects of cyclones or heavy rains and provide higher yield. Introgression of SCM2 in Swarna and Indra reported by Girijarani et al., (2015a)

NIL of Cottondora sannalu, DST 8-162-4 showed highest genome recovery of $91.0 \%$. Above NIL with targeted trait of salinity in the background of highly adaptive variety Cottondora sannalu would perform better in coastal saline soils. Similarly, Hoque et al., (2015) introgressed Saltol QTL into the genetic background of BRRI dhan49 using FL 478 as a donor parent through marker-assisted backcrossing and used 56 polymorphic markers for background selection.

Recently, Nareshbabu et al., (2017) transferred a quantitative trait locus (QTL), Saltol, using FL 478 as donor into Pusa Basmati 1121 through marker assisted backcrossing.

The background genome recovery in the NILs ranged from 93.3 to $99.4 \%$. The improved NILs were either similar or better than the recurrent parent PB1121 for yield, grain and cooking quality and duration.

Among the 24 entries evaluated, NIL MTU 2336-62-25-38-16 of Pushyami, MTU 2244119-59-63-40, MTU 2244-119-83-65 of Amara were identified as NILs with targeted trait Sub1 possessing maximum recovery of respective parents. NILs of Swarna MTU 2546A-34-1-9-1, Indra MTU 2547A-78-19-11, MTU 2547A-95-1-11-1 and Cottondora sannalu MTU 2251A-136-11-1 exhibited maximum genome recovery of respective recurrent parent besides possessing targeted loci SCM2 conferring lodging resistance. Only one NIL of Cottondora sannalu, DST 8162-4 with Saltol loci developed as best line with more genome recovery of recurrent parent.

\section{Agro morphological characterization}

Summary results of morphological distinguished characters among NILs and their respective parents are presented in Table 6.

NIL of Pushyami MTU 2336-70-46-25-44 for Subl showed dark green intensity of leaf colour, medium leaf senescence can be differentiated with recurrent parent Pushyami possessing light green intensity of leaf colour and early leaf senescence. Indentified best NIL of Amara MTU 2244-119-59-63-40 for Subl exhibited weak pubescence of blade surface, medium leaf senescence, medium gelatinizing temperature can be differentiated with recurrent parent Amara possessing medium pubescence of blade surface, early leaf senescence, low gelatinizing temperature.

Identified best NIL of Swarna MTU 2546A34-1-9-1 for SCM2 showed medium pubescence of blade surface, thick stem thickness and medium leaf senescence can be differentiated with recurrent parent Swarna for absence in pubescence of blade surface, thin stem thickness, early leaf senescence. NIL of popular variety Indra MTU 2547A-7819-1-1 for SCM2 showed exhibited no variation in all the agro morphological characters studied with the recurrent parent Indra. 
NIL of Cottondora sannalu DST 8-162-4 for Saltol showed strong pubescence of blade surface, thick stem thickness, long panicle length, medium panicle number per plant $(>11)$ can be differentiated with recurrent parent Cottondora sannalu possessing weak pubescence of blade surface, medium stem thickness, medium panicle length, low panicle number per plant $(<11)$. NIL of Cottondora sannalu MTU 2251A-136-11-1 for SCM2 exhibited strong pubescence of blade surface, thick stem thickness can be differentiated with recurrent parent Cottondora sannalu for weak pubescence of blade surface, medium stem thickness.

In the present investigation, molecular markers showing PIC value $>0.5$ can be used to distinguish NILs from their respective parents apart from gene linked markers of targeted trait. Best NILs with maximum genome recovery of recurrent parent along with morphological distinguished characters were identified.

\section{References}

Ahmed, F., Rafii, M.Y., Ismail, M.Z., Abdul, S.J., Rahim, H.A., Tanweer, F.A. and Latif, M.A. 2016. Recurrent parent genome recovery in different populations with the introgression of Sub1 gene from a crossbetween MR219 and Swarna-Sub1.Euphytica.207:605618.

Anderson, J.A., Churchill, G.A., Autrique, J.E., Tanksley, S.D. and Sorrels, M.E. 1993. Optimizing parental selection for genetic maps.Genome.36:181-186.

Ara, A., Uddin, A.B.M.A., Iftekharuddaula, K.M., Saikat, M.M.H. and Khan, M.A.I. 2015. Introgression of Subl QTL into a rainfed lowland rice varietyof Bangladesh using marker-assisted backcross approach. International Journal of Research. 2 (5): 233-244.
Chakravarthi, B.K. and Naravaneni, R. 2006. SSR marker based DNA fingerprinting and diversity study in rice (Oryza stativa L.). African Journal of Biotechnology. 5: 684-988.

Girijarani, M., Satyanarayana, P. V., Suryanarayana, Y., Ramana rao, P.V., Neerajakshi, CH., Chamundeswari, N., Ravikumar, B.N.S.V.R., Pavani, S.L., Kondayya, K., Ratnasree, P., Vishnuvardhan, K.M., Sivaramprasad, K. and Reddy, A.V. 2015b. Enhancement of flood tolerance in a high yielding rice variety 'amara' by marker assisted selection. SABRAO Journal of Breeding and Genetics, 47 (4): 439-447.

Girijarani, M., Satyanarayana, P.V., Chamundeswari N, Ravi Kumar, B.N.V.S.R., Ramana Rao, P.V., Lalitha Pavani, Kondayya, K., Naga Kumari, P and Jaya Raj, K. 2015a. Development of lodging resistant advanced back cross lines of rice using marker assisted backcross breeding. International Rice Symposium, IIRR, Hyderabad. 18-20 November, 2015. pp: 4.

Gregorio, G.B. 1997. Tagging salinity tolerance genes in rice using amplified fragment Length polymorphism (AFLP). Ph.D. Thesis, University of the Philippines Los Baños. Laguna, Philippines.

Hoque, A.B.M.Z., Haque, M.A., Sarker, M.R.A. and Rahman, M.A. 2015. Marker-assisted introgression of Saltol locus into genetic background of BRRI Dhan-49. International Journal of Biosciences. 6: 71-80.

Hospital, F. and Charcosset, A. 1997. Markerassisted introgression of quantitative trait loci. Genetics. 147:1469-1485.

Iftekharuddaulaa, K.M., Ahmed, H.U., Ghosal, S., Amin, A., Moni, Z.R. Bisnu, P. R., Hirendra, N. B., Siddiquea, M. A., Collard, B.C.Y and 
Septiningsih, E. M. 2016. Development of early maturing submergence-tolerant rice varieties for Bangladesh. Field Crops Research.6594: 10.

Islam, A.S.M.F., Ali, M.R., Gregorio, G.B. and Islam, M.R. 2012. Genetic diversity analysis of stress tolerant rice (Oryza sativa L.). African Journal of Biotechnology.11 (85): 15123-15129.

Kuleung, C., Baenziger, P.S. and Dweikat, I. 2004. Transferability of SSR markers among wheat,rye, and triticale.Theoretical and Applied Genetics. 108: 1147-1150.

Nandi, S., Subudhi, P.K., Senadhira, D., Manigbas, N.L., Sen-Mandi, S. and Huang, N. 1997. Mapping QTL for submergence tolerance in rice by AFLP analysis and selective genotyping. Molecular and General Genetics. 255:1-8.

Nareshbabu, N., Gopala, K.S., Vinod, K.K., Krishnamurthy, S.L., Vivek K.S., Madan P.S., Renu, S., Ranjith K.E., Vandna, R., Haritha, B., Prolay, K., Bhowmick, A.K., Yadav, M.N., Nagendra, K.S, Kumble, V.P. and Singh, A.K. 2017. Marker aided incorporation of Saltol, a major QTL associated with seedling stage salt tolerance, into Oryza sativa 'Pusa Basmati 1121'. Frontiers in Plant Science.8: 41.

Ndour, D. 1998. Tests of Agro-morphological characterization andgenetics of salt tolerance in rice (Oryzasativa L.) in the SenegalRiver Delta. Memory Master II, University Cheikh Anta Diop in Dakar, pp. 1-27.

Ookawa, T., Hobo, T., Yano, M., Murata, K., Ando, T., Miura, H., Asano, K., Ochiai, Y., Ikeda, M., Nishitani, R., Ebitani, T., Ozaki, H., Angeles, E.R., Hirasawa, T. and Matsuoka, M. 2010. New approach for rice improvement using a pleiotropic QTL gene for lodging resistance and yield. Nature Communications.1: 132.

Patel, A.A., Gunvantsinh, C.J., Patel, T.R. and Harshvardhan N. Z. 2016. Study of genetic diversity of Near Isogenic Lines of rice using RAPD markers. Journal of Pure And Applied Microbiology. 10 (1): 799-809.

Renu Singh, Yashi Singh, Suchit Xalaxo, Verulkar, S., Neera Yadav, Shweta Singh, Nisha Singh, Prasad, K.S.N., Kondayya, K., Ramana Rao, P.V., Girija Rani, M., Anuradha, T., Suraynarayana, Y., Sharma, P.C., Krishnamurthy, S.L., Sharma, S.K., Dwivedi, J.L., Singh, A.K., Singh, P.K., Nilanjay, Singh, N.K., Rajesh Kumar, Chetiah, S.K., Ahmad, T., Raii, M., Perraju, P., Anita Pande., Singh, D.N., Mandal, N.P., Reddy, J.N., Singh, O.N., Katara, J.L., Marandi, B., Swain, P., Sarkar, R.K., Singh, D.P., Mohapatra, T., Padmawathi, G., Ram, T., Kathiresan, R.M., Paramsivam, K., Nadarajan, S., Thirumeni, S., Nagarajan, M., Singh, A.K., Prashant Vikram, Arvind Kumar, Septiningshih, E., Singh, U.S., Ismail, A.M., Mackill, D. and Singh, N.K. 2015. From QTL to variety-harnessing the benefits of QTLs for drought, flood and salt tolerance in mega rice varieties of India through a multi-institutional network. Plant Science. http://dx.doi.org/ 10.1016/j. plantsci.2015.08.008.

Rohlf, F.J. 2000. NTSYS-PC, numerical taxonomy and multivariate analysis system. Version 2.02, Applied Biostatistical Inc, New York.

Shobharani, N., Subba Rao, L.V. and Viraktamath. 2006. National guidelines for the conduct of tests for distinctness, uniformity and stability: Rice (Oryza sativa L.) Zero draft, Directorate of Rice Research, Rajendranagar, Hyderabad. pp: 7-15.

Sundaram, R.M., Vishnupriya, M.R., Biradar, 
S.K., Laha, G.S., Reddy, G.A., Rani, N.S., Sharma, N.P. and Sonti, R.V. 2008. Marker assisted introgression of bacterial blight resistance in Samba Mahsuri, an elite indica rice variety. Euphytica.160:411-422.

Toojinda, T., Siangliw, M., Tragoonrung, S. and Vanavichit A. 2003. Thai Jasmine rice carrying QTLCh9 (Sub1 QTL) is submergence tolerant. Annals of Botany. 91:225-261.

Van Berloo R. 2008. GGT 2.0: Versatile software for visualization and analysis of genetic data. Journal of Heredity. 99(2): 232-236.

Venuprasad, R., Impa, S.M., Veeresh, G.R.P., Atlin, G.N. and Serraj, R. 2011. Rice near-isogenic-lines (NILs) contrasting for grain yield under lowland drought stress. Field Crops Research.123: 3846.

Virk, P.S., Ford-Lloyd, B.V., Jockson, M.T. and Newbury, H.J. 1995. Use of RAPD for the study of diversity within plant germplasm collections. Heredity. 74: 170-179.

Xu, K. and Mackill, D.J. 1996. A major locus for submergence tolerance mapped on rice chromosome 9. Molecular Breeding. 2:219-224.

Xu, K., Xia, X., Fukao, T., Canlas, P., Maghirang-Rodriguez, R., Heuer, S., Ismail, A.I., Bailey-Serres, J., Ronald, P.C. and Mackill, D.J., 2006. Sub1A is an ethylene response factor-like gene that confers submergence tolerance to rice. Nature. 442: 705-708.

Zheng, K., Subudhi, P.K., Domingo, J., Magantay, G. and Huang, N. 1995. Rapid DNA isolation for marker assisted selection in rice breeding. Rice Genetics NewsLetter. 12:255-258.

Zhu, Y., Qin, G., Hu, J., Wang, Y., Wang, J. and Zhu, S. 2012. Fingerprinting and variety identification of rice (Oryza sativa L.) based on simple sequence repeat markers. Plant Omics Journal. (4): 421-426.

\section{How to cite this article:}

Sudhakar Reddy, B. and Girija Rani, M. 2018. Molecular and Morphological Characterization of near Isogenic Lines Developed for Major Abiotic Stresses of Rice (Oryza sativa L.). Int.J.Curr.Microbiol.App.Sci. 7(01): 2782-2797. doi: https://doi.org/10.20546/ijcmas.2018.701.333 